\title{
„Veränderungen tun weh, müssen aber sein"
}

\begin{abstract}
Fortbildungskongresse haben im Freien Verband Deutscher Zahnärzte eine lange Tradition. Deshalb treffen Veränderungen in dem Bereich nicht immer auf Begeisterung. Dennoch hat Dr. Michael Betz in seiner Funktion als stellvertretender FVDZ-Bundesvorsitzender und Hauptverantwortlicher für die Kongresse für frischen Wind gesorgt.
\end{abstract}

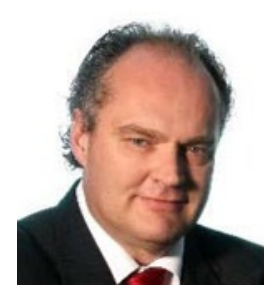

\section{Wo stand der Verband, als Sie den Bereich Kongresse übernommen haben?}

2013 war die Situation so, dass die Fortbildungskongresse des FVDZ bis auf diesen einen gut liefen. Ich habe mir die Kosten der einzelnen Kongresse angesehen und festgestellt, dass der Davoser Winterkongress einfach viel zu teuer ist. Das war zwar schon Jahre zuvor der Fall, aber ganz offensichtlich wollte man mit dieser Tradition nicht brechen und hat das ,,Sorgenkind“ gepflegt, obwohl von Jahr zu Jahr immer weniger Teilnehmer verbucht wurden.

Als ich das erste Mal in Davos dabei war, habe ich auch ganz persönlich gemerkt, wie kostspielig der Aufenthalt war. Spätestens da habe ich für mich gedacht, dass das so nicht bleiben kann.

Darüber hinaus haben wir einige Hauptsponsoren verloren, die wegen der Zollmodalitäten in der Schweiz abgesprungen waren, aber Bereitschaft signalisierten, bei einer Konzeptänderung wieder dabei sein zu wollen. Spätestens da mussten wir wirtschaftlich umdenken und entsprechend handeln.

\section{Was haben Sie in der zurückliegenden Legislaturperiode erreicht?}

Wir haben im Bundesvorstand entschieden, dass wir grundsätzlich an einem Winterkongress festhalten wollen, aber eben nicht mehr in der Schweiz. Inhaltlich sollte sich nicht viel ändern, es war und bleibt ein anspruchsvoller Fortbildungskongress mit einer breiten Palette von hochwertigen Seminaren und Workshops.

Ich habe mir überlegt, welcher Skiort - besonders für junge Kolleginnen und Kollegen - eine attraktive und bezahlbare Location sein könnte. Wo es also neben der Fortbildung ein gutes Freizeitangebot gibt, das auch mehr jüngere Zahnärztinnen und
Zahnärzte und ihre Familien anlockt. Und so bin ich auf Ischgl gekommen. Die Gemeinde in Tirol kenne ich selbst ganz gut. Dort wird mehr geboten als in Davos, und die Preise sind moderater.

Auch terminlich haben wir etwas verändert: Wir sind bewusst von der traditionellen Karnevalswoche, in der der Kongress bislang stattgefunden hatte, in die erste Märzwoche gegangen. Dadurch konnten wir Kosten sparen, weil die Preise in der Karnevalszeit noch mal anziehen. Dies wurde auch von den Kollegen aus den Karnevalshochburgen dankend aufgenommen, waren sie in den Jahren zuvor doch immer wieder im Zwiespalt.

Ich weiß, dass der Standortwechsel nicht überall Begeisterungsstürme hervorgerufen hat. Insbesondere nicht in den Reihen jener, die dem Kongress jahrzehntelang die Treue gehalten haben. Aber wenn einem Fortbildungskongress die Teilnehmer und Sponsoren ausgehen, dann muss man im Sinne des Verbandes handeln und Neues ausprobieren. Veränderungen tun weh, vor allem bei traditionellen Gepflogenheiten. Aber sie müssen manchmal sein - so wie beim Winterkongress.

\section{Was ist das Ziel für die nächste Wahlperiode?}

Ich möchte die Kontinuität bei den Kongressen beibehalten. Mit dem Kongress in Ischgl kann ich mir nicht vorstellen, dass wir eine Bauchlandung hinlegen. Aber wir müssen trotzdem nach einem Jahr ganz ehrlich Bilanz ziehen und sehen, ob sich der Umzug gelohnt hat. Wenn ja, dann wird es ein wesentlicher Baustein unseres Fortbildungsangebotes werden.

Die anderen FVDZ-Kongresse sind eigentlich Selbstläufer. Der Usedomer Sommerkongress läuft genauso hervorragend wie der Praxis-Ökonomiekongress auf Sylt. 